terms of the variability of June-September (JJAS) rainfall averaged over the whole subcontinent, all of the CMIP5 models fall within the range of variability, so all pass the test of being a 'good' model by this measure.

No climate modeller would believe that their model is a perfect representation of the climate system. A similar analysis to Fig. 1 for the ability of the CMIP5 models to reproduce the mean and variability of the South Pacific Convergence Zone would show that all models fail to reproduce the position of that feature with absolute fidelity. Nevertheless, for some aspects of the climate, some models are now falling within the range of observational uncertainty. The situation is more acute when we attempt to evaluate processes where the only observational estimates are from special field campaigns, point measurements or from re-analysis products that blend observations with other model outputs. Is the principal barrier to model improvement a lack of accurate observations rather than model resolution and parameterization of physical processes? Activities such as Obs4MIPs, which collect together observational data sets to use in model evaluation, may help to identify some of these problems, but we believe that the uncertainties in observations will become a more critical issue as climate models improve.

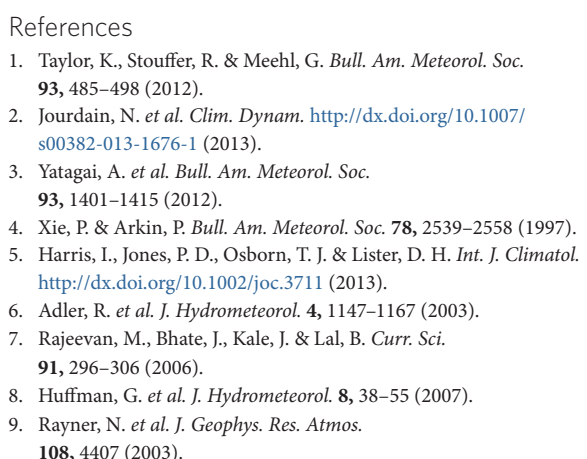

Taylor, K., Stouffer, R. \& Meehl, G. Bull. Am. Meteorol. Soc. 93, 485-498 (2012) 0382-013-1676-1 (2013)

3. Yatagai, A. et al. Bull. Am. Meteorol. Soc

, 1401-1415 (2012)

Harris, I., Jones, P. D., Osborn, T. J. \& Lister, D. H. Int. J. Climatol.

91, 296-306 (2006)

9. Rayner, N. et al. J. Geophys. Res. Atmos.

10. Smith, T. \& Reynolds, R. J. Clim. 16, 1495-1510 (2003).

11. Kaplan, A. et al. J. Geophys. Res. Oceans 103, 18567-18589 (1998).

Acknowledgments

This work was supported by the joint NERC/MoES SAPRISE project (NE/I022841/1). A.T. was also supported by a NERC Fellowship (NE/H015655/1).

Mat Collins ${ }^{1 *}$, Krishna AchutaRao ${ }^{2}$,

Karumuri Ashok ${ }^{3}$, Satyendra Bhandari ${ }^{4}$,

$\square \quad$ Ashis K. Mitra ${ }^{5}$, Satya Prakash ${ }^{5}$, Rohit Srivastava ${ }^{4}$ and Andrew Turner ${ }^{6}$

${ }^{1}$ College of Engineering, Mathetmatics and Physical Sciences, University of Exeter, Exeter EX4 4QF, UK, Indian Institute of Technology Delhi, Delhi 110016, India, ${ }^{3}$ Indian Institute of Tropical Meteorology, Pune 411008, India, ${ }^{4}$ Indian Centre for Climate and Societal Impacts Research, Ahmedabad 380009, India, ${ }^{5}$ National Centre for Medium Range Weather Forecasting, Noida 201309, India, ${ }^{6}$ National Centre for Atmospheric Science-Climate, University of Reading, Reading RG6 6BB, UK. *e-mail:M.Collins@exeter.ac.uk

\title{
Prudence on solar climate engineering
}

To the Editor - The call for prudence by Schäfer et al. (Nature Clim. Change 3,$766 ; 2013$ ) in relation to field tests of solar climate engineering is welcome. We applaud their effort to help establish informal norms to stifle counterproductive experimentation. However, we should be careful to make sure that what is called 'prudence' does not hamper beneficial scientific and technical investigation. Furthermore, efforts to develop more formal governance mechanisms, specifically around solar climate engineering field experiments, may prove wrong-headed. It may be wiser to create mechanisms for governing the more general case of experiments conducted in international waters or airspace that have prima facie potential to produce more than trifling harm to an international commons or across international boundaries.

The phrase 'field test of solar climate engineering' cannot be unambiguously defined. If I paint a one metre square with white paint on my dark asphalt driveway and measure the reflected sunlight, is that a field test of solar climate engineering? It would seem that if my intent were to develop technologies that would ultimately modify climate at the global scale, then the answer would probably be 'yes'. If the aim were simply to test which asphalt paints are easiest on the eye, then the answer would probably be 'no'.

So, whether this is a solar climate engineering field test or not depends not only on my actions, but also on my intent.

But what if someone else is funding this project, and they want to develop a solar climate engineering system but I just want driveway paints that are easier on the eye (or vice versa)? Whose objective counts, that of the experimental scientist or that of the funder?

It is problematic to require that issues of intent be resolved to determine which governance regime applies. In a workable regulatory system, a physical description of the action should determine whether the governance scheme applies. It seems for a governance regime to be triggered, the risks of damage from a project must derive from a physical effect that exceeds some specified minimal level: the intent of the various parties involved in a project should be taken into account when determining whether expected benefits exceed expected damage in any particular case.

Schäfer et al. are correct in suggesting that even such low-impact experiments could provoke a negative and ultimately counter-productive backlash, and therefore they are well justified in counselling scientists and engineers to proceed cautiously and prudently. However, prudence does not always mean refraining from performing an experiment. Galileo's peers may well have felt that he was imprudent in dropping the balls off the leaning tower of Pisa, but that doesn't mean that he shouldn't have dropped them.

The central point is that a governance regime can consider intent in weighing whether an activity can go forward, but determination of intent should not be a prerequisite for determining which scheme of governance applies. The trigger for a formal regime should be based on a physical description of the proposed activities and not its intent.

The governance regime should be responsible for determining intent. It would be impractical to have a 'pregovernance' process to first determine intent so that it can be decided whether a governance regime should apply. How would it be decided which activities are subject to this pregovernance process?

Ken Caldeira* and Katharine L. Ricke Carnegie Institution for Science Department of Global Ecology, 260 Panama Street, Stanford, California 94305, USA.

*e-mail: kcaldeira@carnegiescience.edu 